\title{
Integrated assessment frameworks for understanding pathways for socially inclusive agricultural intensification
}

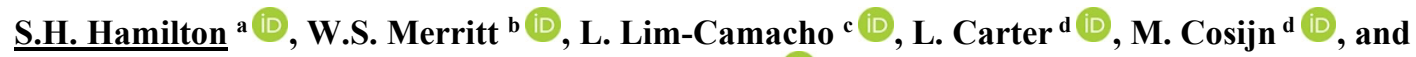 \\ C.H. Roth d (iD) \\ ${ }^{a}$ School of Science, Edith Cowan University, Joondalup, Western Australia, ${ }^{b}$ Fenner School of Environment \\ and Society, The Australian National University, Australian Capital Territory, ${ }^{c}$ CSIRO Agriculture and \\ Food, ${ }^{d}$ CSIRO Land \& Water, Brisbane, Queensland \\ Email: s.hamilton@ecu.edu.au
}

\begin{abstract}
Alleviating poverty through agricultural development is a challenge that requires sound understanding of the social, market, environmental and institutional settings. An integrated assessment (IA) process can help to clarify and examine the nature of the interactions between these diverse processes and facilitate concerted and collaborative efforts from interdisciplinary teams. This paper provides an overview of IA frameworks developed for a project aimed at identifying opportunities and policy options that promote more socially inclusive and sustainable agricultural intensification in rural communities in West Bengal and Bangladesh.

The IA frameworks were intended to provide a 'big picture' of the social and agricultural systems we are researching, and to improve understanding of the interrelationships between the diverse processes, and the pathways between drivers and outcomes. This paper describes the methodological process followed in developing these frameworks (Figure 1). The frameworks are grounded in both theory and observations from project activities, and were iteratively developed with input from stakeholders and domain experts. The frameworks then formed the basis for further (semi)quantitative or qualitative analysis, demonstrated in the study through the development of semi-quantitative models (fuzzy cognitive maps) and narratives.
\end{abstract}

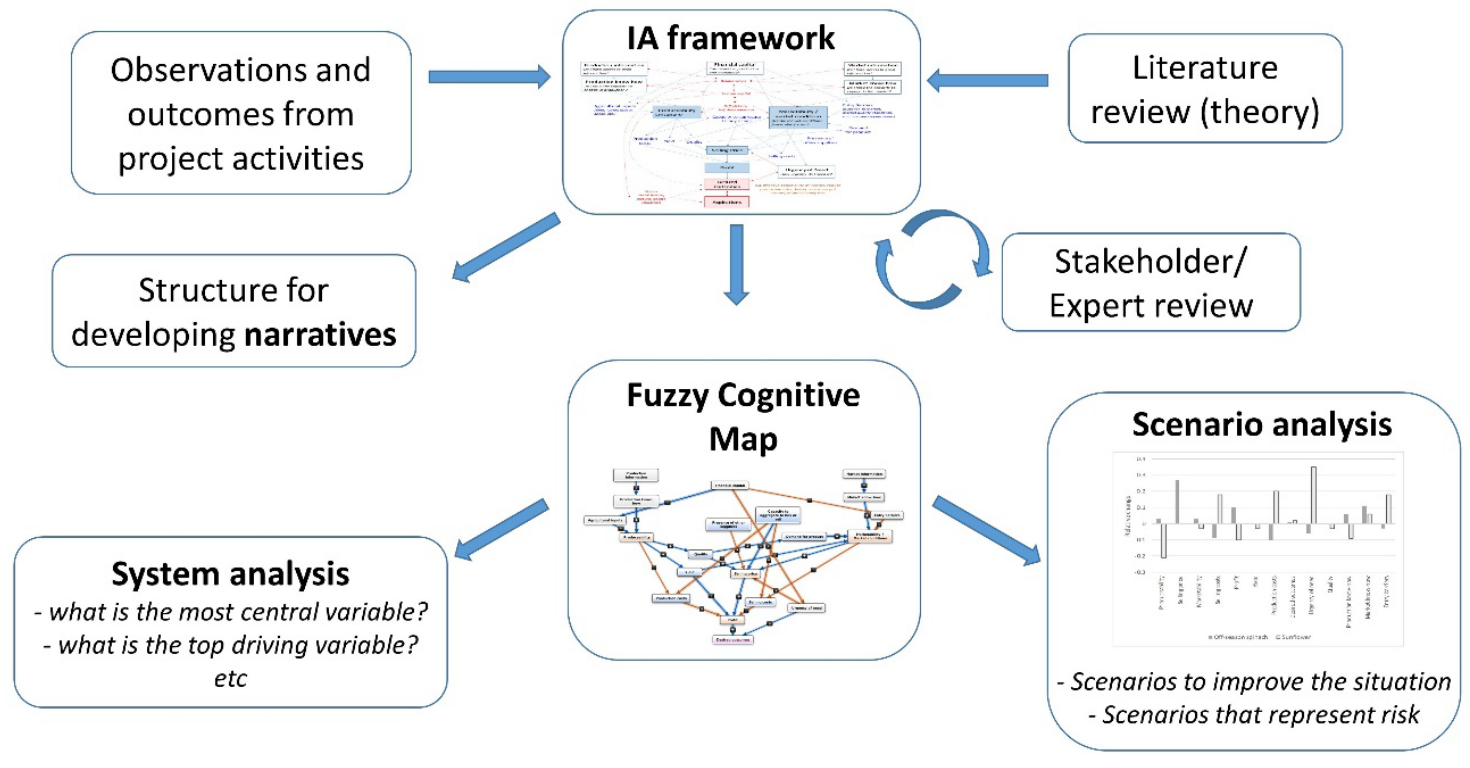

Figure 1. An overview of the integrated assessment process

As a modelling process, an outcome of the IA work has been the formalisation of our common understanding of the system. The IA framework development also facilitated an explicit integration process, as it involved all team members who were required to consider the linkages between their usual fields of study to the wider system, thereby promoting interdisciplinary thinking. The IA frameworks have also become a discussion and learning tool to test implications of system interventions or perturbations.

Keywords: Integrated assessment, interdisciplinary research, modelling process 


\section{INTRODUCTION}

Despite great advances in agricultural production, due to (e.g.) the development and adoption of high-yielding and drought-tolerant crop varieties, irrigation infrastructure and improved cultivation methods, a vast number of rural communities across the globe are not benefitting from these technologies. Even with the presence of opportunities, for example through development aid interventions, benefits may be limited within the community to large-holder or more affluent farmers. Marginal groups, such as small-holder, landless and women farmers and tribal communities, often face serious constraints that prevent them engaging in interventions and improving their situation. To meaningfully address such problems in international development, research needs to go beyond the focus on the biophysical to understand the social and institutional intricacies and, critically, system interactions.

These international development problems contain all the elements of a "wicked problem", whereby there is a high degree of uncertainty, cause-effect relations are complex and tangled, and solutions are unclear. Such problems require a concerted, collaborative effort of all affected parties (Parrott 2017), including the community, policy makers and scientists. Modelling can contribute to this process as a tool for organising data, knowledge and assumptions, and facilitating some of the dialogue between parties. Integrated assessment (IA) models can combine multiple and diverse components, crossing disciplinary, organisational and conceptual boundaries, to provide a broader picture of the problem and system.

This paper presents an overview of a series of IA frameworks developed for the project, Promoting Socially Inclusive and sustainable Agricultural Intensification in West Bengal and Bangladesh, herein referred to as "SIAGI" (www.siagi.org). The project has explored the various social, market, environmental and institutional issues and processes involved with agricultural intensification in six rural communities in West Bengal (India) and Bangladesh. Particular focus has been on understanding how to address the issues faced by the marginalised and disadvantaged groups who are at risk of social exclusion and increasing inequity. The IA frameworks aimed to provide a 'big picture' of the system and understand the interrelationships between the diverse issues and processes, and the pathways between drivers and outcomes. The IA frameworks are intended as discussion support tools for assessing interventions and identifying opportunities in these communities, including understanding the conditions under which interventions can be effective.

\section{PROJECT BACKGROUND}

The SIAGI project aims to understand the risks of agricultural intensification in rural communities in West Bengal and Bangladesh, and to identify opportunities and policy options that promote more socially inclusive and environmental sustainable intensification. Two of the four West Bengal study sites are located in the northern eastern Indo Gangetic Plains and on the East India Plateau, respectively, while two other sites are located along the coastal zones of southern Bangladesh. An overview of the general factors influencing agricultural livelihoods across the case studies is provided in Section 3.

The SIAGI project worked alongside two other 'sister' projects - all three projects funded by the Australian Centre for International Agricultural Research (ACIAR). The sister projects were focussed on the provision of irrigation to the villages. Other interventions involved building of capacity in farmers through training and demonstrations in production and marketing, and exposure visits to farms in other villages. Through our partner NGOs, who engaged directly with the communities, we also encouraged the formation of farmer collectives and connections with other groups (i.e. building social capital).

With an interdisciplinary team of over 40 people, this large project adopted several research methods for obtaining and analysing information, including community engagement processes, value chain analysis, policy analysis, institutional mapping, bio-economic modelling and integrated assessment modelling. The IA modelling has drawn on outputs and insights from these other research activities, and in turn informed their further development. IA modelling has served as one of the synthesis tools for the project.

This paper builds on Hamilton et al. (2017) where we presented a generic conceptual framework to elicit and communicate understanding about complex problems in the first phase of the IA modelling process of SIAGI. That earlier work was focussed on providing a common language to overcome challenges associated with working in interdisciplinary teams - i.e. differences in terminologies, theories, methods, perspectives and interests. The resulting applications helped to start capturing the key elements and processes of system and mapping out the pathways of influence between drivers of change and impacts on communities. Since then, through the various project activities, the team has developed a deeper understanding of the key issues and processes in the villages. 


\section{OVERVIEW OF CONSTRAINTS AND OPPORTUNITIES IN AGRICULTURE}

This overview summarises the key observations of small and marginal farmers across the case study communities, stemming from research activities for the larger SIAGI project. See https://siagi.org/projectresources/ for reports containing more detailed information on the various topics.

Small and marginal farmers in our case study villages are commonly faced with difficulties in achieving adequate agricultural production to meet their various household needs, including income, nutrition, education, health, and comfort. Achieving sustainable agricultural intensification is often considered a central means of improving the social and economic conditions of these households. Opportunities for agricultural intensification may involve higher use of agricultural inputs, water resource development for irrigation, the adoption of technology or better practices (on-farm or post-harvest), and switching to higher value, higher yielding or drought-tolerant crop varieties. The challenge is in ensuring that small and marginalised farmers are not excluded from, or disadvantaged by, agricultural intensification. A wide range of, often interrelated, constraints at multiple levels (from individual, household, and community, through to state and national) need to be overcome for socially inclusive and sustainable intensification to occur in the communities.

The impact of increased climate variability on agricultural production is common across the case studies. The villages are susceptible to climate risks and variability that affect access to water, and climate change is expected to increase the likelihood and intensity of these risks. This environmental context and its associated vulnerability is intrinsically linked with both institutional water management and community irrigation management. In the two Bangladesh villages, institutional water management is posed in terms of salinity intrusion and thus freshwater availability in the dry season. In the West Bengal study villages, freshwater availability for agriculture in the dry season is largely determined by access to stored surface water or groundwater pumps.

Small and marginal farmers in our study communities often receive low prices for their produce, especially at times of oversupply or when obliged into 'distressed selling' because of financial burden. This may be alleviated by the farmers making crop choices based on market factors (i.e. selecting high value crops) or changing their marketing practices. Opportunities exist for value adding post-harvest, for example, graded, sorted, cleaned or milled produce may fetch higher prices or open up new markets (e.g. graded produce can be sold to processing factories). Other opportunities for increasing value include off-season production (e.g. through greenhouse production) and off-season selling through storage of produce at times of oversupply.

Low profitability for small and marginal farmers can also stem from the low production volumes that do not offset the cost of production and trading. Farmers may get better prices when their produce is sold at the market, rather than at farm gate, however transport and other transaction costs make selling at market prohibitive for some farmers. Collectives and Farmer Producer Organisations may provide small farmers the opportunity to improve their economies of scale in this regard, as well as remove market entry barriers such as minimum supply volumes (Bijman et al., 2011).

The costs of agricultural technology and inputs can be a major barrier for many small and marginal farmers in achieving higher income. This is exacerbated by a lack of income certainty from climate risks and market price fluctuations. Without the financial capital to invest in the farm, they risk reinforcing the cycle of poor production and low income. Some opportunities exist in getting support from external agencies, with many public policies aimed at promoting agricultural intensification. However, in both India and Bangladesh, the institutional arrangements have weaknesses that result in inefficient and inequitable access to resources and services.

Small and marginalised households often do not access government resources or services, partly due to lack of awareness of the schemes or limited capacities to initiate access to schemes. For example, some people may be discouraged from applying for a scheme if a large amount of paperwork is required, particularly if they are illiterate. In addition, many small and marginalised (including women-headed) farmers lack agency to make changes in their lives, and are reluctant to seek advice or help. Another key social issue in all of our study communities is out-migration caused by lack of profitability in agriculture or lack of local work year-round, which forces many men to seek income elsewhere. This places high work pressure on the women left to tend the farm and look after the family (i.e. feminisation of agriculture) (Pattnaik et al. 2017). At a village level, the seasonal migration also leads to labour shortage particularly during the dry season.

Given the interwoven nature of issues related to social inclusion and agricultural intensification, it is crucial that interventions and policies are not too narrowly focussed, are multi-faceted, and are attentive to the context of the people they intend to help. 


\section{THE FRAMEWORK DEVELOPMENT PROCESS}

The constraints and opportunities for marginalised households in agricultural intensification, described above, were captured in a series of IA component frameworks. The frameworks are grounded in both theory and observations from SIAGI and its sister projects. The development of each framework is a culmination of workshops, discussions with project team members, community visits and literature reviews, through which they were iteratively developed, reviewed and revised.

Within each component framework, we identify and link the key elements and causal pathways of the processes involved. For example, the value chain component represents all important factors related to growing and selling the product, and how they relate to one another and to the desired outcomes of the farmer. The framework maps out assumptions about the entire process of change, from interventions or activities through to the targeted outcomes; this includes the intermediate outcomes and the set of conditions that enable the change. This articulation of the connection between activities and outcomes is akin to Theory of Change (Weiss, 1995).

The framework takes the perspective of an individual farmer or farmer group (referred to as the actors) when defining objectives and outcomes. In doing so, we look beyond the generalised outcomes (e.g. increased income) that are normally presumed or determined by external bodies, and recognise that individuals may have their own desired outcomes (e.g. good education for their children, or reduced workload). The principles that helped guide the design of the frameworks are summarised in Figure 2. Interventions have intermediate outcomes (a) that lead to generalised outcomes (b), which subsequently result in the desired outcomes (c) specific to the actors and their aspirations. The progressive outcomes are dependent on a number of conditions and are bounded by the contextual factors of the actor(s) including their location (which dictates the environmental setting,

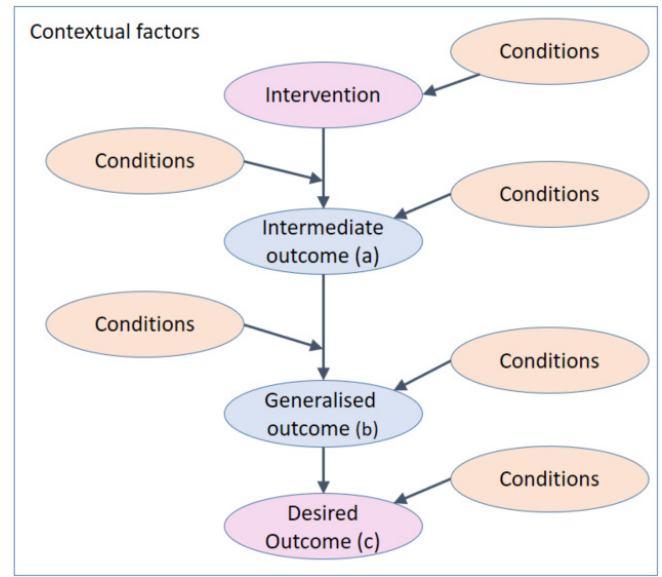

Figure 2. Guiding principles distance to markets, etc), and their socio-cultural, political and economic setting and positioning. The frameworks are generic in that they can be applied to different contexts (i.e. across SIAGI case studies and beyond). However, the state of each element and the strength of relationships between them varies between contexts (e.g., cultural, socioeconomic, and environmental setting).

The first drafts of the frameworks were developed in separate workshops involving the modellers and at least one domain expert from the project team. The workshop process involved intensive discussion on the key elements of the theme and their casual connections, often beginning with a few key concepts as central building blocks (following Murungweni et al. 2011). For example, the value chain framework (shown in Fig. 4) started with 'access to market information' and 'improved income' as the central building blocks, whereas the empowering change framework started with 'relationships', 'self-efficacy', 'social capital', 'empowerment', and 'social norms' as its initial building blocks. The key variables (or concepts), relationships, and other relevant factors that bridged the central building blocks were then identified and discussed. The workshop discussion drew on anecdotes and observations from the case study villages.

After the workshop process, the key concepts identified were further researched to ensure the definitions and notions around each were in line with the international literature. The literature also invoked ideas on how to better frame or structure many of the concepts and relationships. An updated framework was then discussed with workshop participants, and revised according to feedback. The framework, along with accompanying text that defined each concept and described each relationship, was then presented to the wider project team for review and comment, and subsequently revised. This review and revision process, occurred over many cycles for each framework. The robustness of the frameworks was then tested by applying them to various scenarios (e.g. different crops, interventions, climate) across study villages and contexts, to ensure they represent the diversity of values and outcomes in the communities. 


\section{OVERARCHING INTEGRATED ASSESSMENT FRAMEWORK}

Three main themes emerged through a workshop process with the project team, upon which the component frameworks were based. Figure 3 provides an overview of the overarching framework, and its three interacting components, summarised as:

i) Local water management - this component focuses on how local water governance and community engagement affect available freshwater resources.

ii) Inclusive Value Chain Analysis - this component assesses the factors determining the produceability and marketability of a product, and its subsequent links to the desired outcomes of farmers, which is often but not limited to income-related outcomes.

iii) Empowering change - this component represents the psychosocial processes related to behavioural change that underlie other components. Empowerment is treated as a process of change which we examine through pathways of motivation, selfefficacy, agency and access to resources.

Each component framework contains a network of key elements and processes related to each theme (for example see Figure 4a). A detailed description of each framework is beyond the scope of this paper.

\section{FRAMEWORK APPLICATION}

The frameworks formed the basis for further (semi)quantitative or qualitative analysis, demonstrated in the study through the development of semi-quantitative models (fuzzy cognitive mapping, FCM) and narratives. The framework provides a structure (i.e. identifies key variables and relationships) upon which narratives can be developed. This structured approach can help ensure information is collected systematically without missing important details, and can also enable better comparison between cases. For the water management and value chain applications, case study narratives based around the frameworks were firstly drafted by the modellers using existing reports and observations. Identified gaps in the narratives were filled by discussions with the onground team, who consulted with the community if necessary. For the empowering change application, since information from the community around the topic had not been documented to the extent of value chain and water management, which were the subject of other project activities, narratives were elicited directly from the community. Questions were asked about how and why the farmer changed, and intended to evoke anecdotes that trace the journey of farmers from before the change, to the events or conditions that triggered the change, to the outcomes of the change and whether the change has been sustained. The framework itself was not presented to farmers and we avoided leading questions that described the relationships in the framework.

For the semi-quantitative analysis, the framework formed the basis of the model structure. FCM was selected as the modelling approach due to its flexibility in that concepts can represent any characteristic or factor, and its intuitive structure and method in quantifying relationships (Ozesmi and Ozesmi 2004). The direction and strength of relationships in FCM are specified as weights between -1 and +1 , which can be elicited from experts or stakeholders. The relative simplicity of the approach deems it suitable for participatory modelling and working with interdisciplinary teams (Diniz et al. 2015; Halbrendt et al., 2014). The model was developed in Mental Modeler (Gray et al., 2013; www.mentalmodeler.org/), with the structure based on the framework and the strength of cause-effect linkages defined and reviewed by the project team. The resultant FCM was analysed to provide measures of the variables in the system, including their centrality scores (relative importance in the network) and their in- and out-degree scores (strength of incoming and outgoing connections). The FCM were also used to test scenarios, exploring possible effects of interventions and system perturbations.

The narratives and quantitative analyses complement one another. The example in Figure 4 shows the value chain IA framework, its FCM, and one of the ways that narratives have been used in this study. In Figure 4c the 'produceability' submodel of the FCM is shown along with the relative states and narratives for a case study on off-season spinach in the West Bengal village of Dhaloguri. 


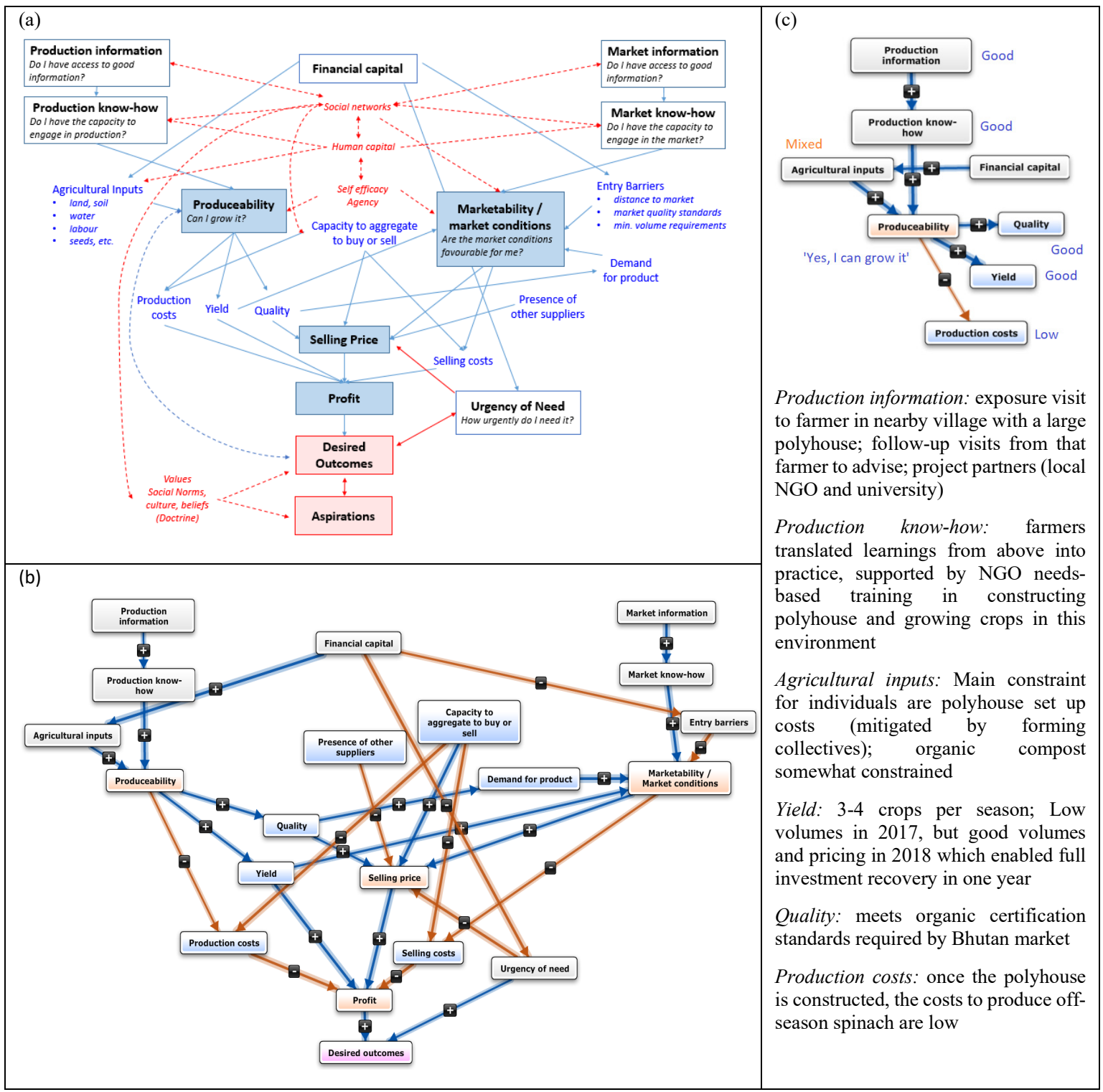

Figure 4. An example of one of the Integrated Assessment frameworks and its application. The inclusive value chain IA framework (a), its application in Fuzzy Cognitive Mapping (b), and examples of narratives used to describe relative states of a subset of the FCM for a case study on off-season spinach in Dhaloguri, West Bengal.

\section{DISCUSSION AND CONCLUSION}

Our integrated assessment work has had two distinct outcomes: 1) frameworks to formalise our understanding of the system(s) we are dealing with; and 2) a discussion and learning tool to test implications of system interventions or perturbations. The frameworks have been a tool to synthesize and distil key information gathered through the various project activities of SIAGI and its sister projects. While some processes or relationships were straightforward, many others required further investigation and extensive consultation with the community and/or domain experts within the project team. The frameworks capture our understanding to date of the system, and are subject to change as we learn more about the system.

Given the interdisciplinary nature of the framework themes, developing the frameworks entailed integration of data, information and perspectives across multiple fields of study and from the community, practitioners and researchers. All members of the project team were involved to some extent in the development of the frameworks, either through the provision of input, review of the frameworks or direct involvement in identifying and qualifying the key elements of the framework. Involvement in the development or review of these frameworks, required team members to consider the linkages between their usual fields of study to the wider system, thereby promoting interdisciplinary thinking. 
To cope with the complexity of the system, the framework was broken into the three thematic components. We found that with the vast number of concepts and links, a single framework that attempted to capture the wholeof-system at detail was considered too complicated (or viewed as a "horrendogram"). This was the case even if each concept and link was simple to understand. This made whole-of-system models or frameworks (as in Hamilton et al. 2017) difficult to obtain feedback on and use for engagement. The component frameworks on the other hand, enabled us to more easily engage team members and stakeholders about their respective domain area and elicit input and feedback at greater detail.

The component frameworks are intended as a discussion and learning tool for project teams to test ideas around agricultural interventions and policies. They allow researchers and development actors to reflect upon the implications an intervention or policy could have on the broader social and economic system surrounding an actor (i.e. the farming community) and the conditions and contexts that will enable positive outcomes to be achieved. This could potentially be helpful to all stages of a project or intervention from the design to completion, and its monitoring and evaluation. By helping to conceptualise and structure the processes of change and impact pathways, the frameworks may help provide clarity on what evidence (e.g. measured indicators) can be used or sought to evaluate past or future project outputs. As a Monitoring, Evaluation and Learning (MEL) tool, the frameworks can be used to assess whether or not a project or intervention is tracking towards desired outcomes, and whether unintended outcomes or consequences may take place. With respect to project design, the framework can help ensure all important factors are considered, so that significant constraints are appropriately addressed before implementation. It is possible that certain scenarios will require a suite of interventions, rather than singular actions and as such will require a broader range of project resources. It may be that certain constraints cannot be reasonably surmounted within the project timeframe or available resources, in which case the intervention may be deemed unfeasible. In such cases, by helping to identify these constraints, the framework can guide allocation resources towards lower risk interventions or activities. It is intended that the component frameworks be used in conjunction to explore how the system works and the pathways through which interventions work and potential constraints to their impact.

\section{ACKNOWLEDGMENTS}

This project (LWR/2014/072) is funded by the Australian Centre for International Agricultural Research (ACIAR). The authors acknowledge contributions from all members of the SIAGI project team.

\section{REFERENCES}

Bijman, J., Muradian, R. Cechin, A. 2011. Agricultural cooperatives and value chain coordination. Value chains, social inclusion and economic development: contrasting theories and realities. Routledge.

Diniz, F.H., Kok, K., Hoogstra-Klein, M.A., Arts, B. (2015). Mapping future changes in livelihood security and environmental sustainability based on perceptions of small farmers in the Brazilian Amazon. Ecology and Society, 20(2).

Gray, S. A., Gray, S., Cox, L. J., Henly-Shepard, S. (2013). Mental modeler: a fuzzy-logic cognitive mapping modeling tool for adaptive environmental management. System sciences (hicss), 2013 46th Hawaii international conference on, 2013. IEEE, 965-973.

Hamilton, S.H., Merritt, W.S. Carter, L, Lim-Camacho, L. Nidumolu, U., Cosijn, M., Mishra, R., Dash, M., Roth, C. (2017). Developing a common language for transdisciplinary modelling teams using a generic conceptual framework. MODSIM 2017, 22nd International Congress on Modelling and Simulation. Modelling and Simulation Society of Australia and New Zealand, December 2017, pp. 1447-1453.

Halbrendt, J., Gray, S.A., Crow, S., Radovich, T., Kimura, A.H., Tamang, B.B. (2014). Differences in farmer and expert beliefs and the perceived impacts of conservation agriculture. Global Environmental Change, 28, 50-62.

Murungweni, C., Van Wijk, M.T., Andersson, J.A., Smaling, E.M.A., Giller. K.E. (2011). Application of fuzzy cognitive mapping in livelihood vulnerability analysis. Ecology and Society 16(4): 8.

Özesmi, U., Özesmi, S.L. (2004). Ecological models based on people's knowledge: a multi-step fuzzy cognitive mapping approach. Ecological Modelling 176, 43-64.

Parrott, L. (2017). The modelling spiral for solving 'wicked' environmental problems: guidance for stakeholder involvement and collaborative model development. Methods in Ecology and Evolution 8(8), 1005-1011.

Pattnaik, I., Lahiri-Dutt, K., Lockie, S., Pritchard, B. (2017). The feminization of agriculture or the feminization of agrarian distress? Tracking the trajectory of women in agriculture in India. Journal of the Asia Pacific Economy 23, doi.org/10.1080/13547860.2017.1394569.

Weiss, C.H. (1995). Nothing as practical as good theory: exploring theory based evaluation for comprehensive community initiatives for children and families. In: Connell et al. (ed) New Approaches to Evaluating Community Initiatives: Concepts, Methods, and Contexts. Washington, DC: Aspen Institute. 